\title{
Carboxymethyllysine and carboxyethyllysine in multiple sclerosis patients
}

\author{
Aleksandra Damasiewicz-Bodzek¹, Beata Łabuz-Roszak²,3, Bartłomiej Kumaszka4, \\ Krystyna Tyrpień-Golder ${ }^{1}$
}

\author{
${ }^{1}$ Department of Chemistry, School of Medical Sciences in Zabrze, Medical University \\ of Silesia in Katowice, Poland \\ ${ }^{2}$ Department of Basic Medical Sciences, School of Health Sciences in Bytom, Medical \\ University of Silesia in Katowice, Poland \\ ${ }^{3}$ Department of Neurology, Provincial Specialist Hospital, Opole, Poland \\ ${ }^{4}$ Prelate of J. Glowatzki District Hospital, Strzelce Opolskie, Poland
}

Submitted: 14 February 2020

Accepted: 19 April 2020

Arch Med Sci

DOI: https://doi.org/10.5114/aoms.2020.95654

Copyright () 2020 Termedia \& Banach

\begin{abstract}
Introduction: Advanced glycation end-products (AGE) are involved in the pathogenesis of many diseases, including neurodegenerative diseases such as multiple sclerosis (MS). The aim of the study was to evaluate the intensity of the protein glycation process in patients with multiple sclerosis and its possible involvement in disease activity.

Material and methods: The study group $(n=45)$ consisted of patients suffering from MS, and the control group $(n=31)$ consisted of healthy adults. Concentrations of selected glycation markers such as carboxymethyllysine $(\mathrm{CML})$ and carboxyethyllysine (CEL) in sera of patients with MS and healthy volunteers were determined by enzyme-linked immunosorbent assay (ELISA). Results: Serum CML and CEL concentrations in patients with MS were higher than in healthy volunteers but only for CML the difference was statistically significant. CML concentrations positively correlated with CEL concentrations only in the healthy persons. In MS patients the serum CML and CEL concentrations did not differ significantly depending on the duration of the disease and depending on the EDSS (Expanded Disability Status Scale) score.

Conclusions: Multiple sclerosis is accompanied by an intensification of protein glycation processes, especially within the pathways leading to the formation of carboxymethyllysine. The duration of the disease and the degree of motor impairment do not appear to affect the progression of the glycation processes. However, the disease process associated with multiple sclerosis may affect the relationship between CML and CEL concentrations.
\end{abstract}

Key words: advanced glycation end-products, multiple sclerosis, carboxymethyllysine, carboxyethyllysine, ELISA.

\section{Introduction}

Multiple sclerosis (MS) belongs to the group of chronic autoimmune inflammatory diseases of the central nervous system. Morphologically, it is characterised by inflammation, demyelination, astrogliosis, oligodendropathy, and neurodegeneration [1-5]. The aetiopathogenesis of the disease is not fully understood. Infectious, genetic, environmental, and immune factors are known to be involved [6-15]. In the last decades, vitamin $D$ deficiency has been hypothesised as one of the fac-
Corresponding author:

Aleksandra DamasiewiczBodzek

Department of Chemistry

School of

Medical Sciences

Medical University

of Silesia

Katowice, Poland

E-mail: aleksandra.bodzek@

sum.edu.pl 
tors that influences the risk of MS [16-20]. It has been suggested that mechanisms dependent on free radicals, imbalance in the antioxidative system, and advanced glycation of proteins may also be involved in the development of this disease [21]. Low molecular weight advanced glycation end-products (AGEs) formed and circulating in the body as a result of the Maillard reaction and those delivered to the body with food (gliotoxins) are removed from the bloodstream by lysosomal degradation via receptors located on the surface of cells. However, most of them are not degraded and accumulate in the body. Their excessive accumulation on cytoplasmic and serum protein, lipid, and nucleic acid molecules leads to oxidative stress and carbonyl stress and the formation of anti-AGE antibodies and cell destructive processes. In addition, AGEs lead to changes in the spatial conformation of proteins and hence the loss of the original properties of these molecules and the disruption of their functions [22-24]. The most common AGEs are $\mathrm{N}^{\varepsilon}$-carboxymethyllysine $(\mathrm{CML})$, $\mathrm{N}^{\varepsilon}$-carboxyethyllysine (CEL), $\mathrm{N}^{\varepsilon}$-lactatolysine, pyrraline, pentosidine, and imidazoles. However, there are many other end-products with unidentified structure and origin [25-29]. CEL is formed by the reaction of methylglyoxal (MGO) with lysine, whereas CML by the reaction of glyoxal (GO) with lysine. Both $\mathrm{MGO}$ and $\mathrm{GO}$ are major precursors in the formation of AGEs that are formed during glycolysis into which the cell metabolism is switched [30]. Glycation and oxidation of proteins are processes that occur physiologically in the body. However, they can also cause adverse changes in tissues and organs, especially when these processes occur under pathological conditions (e.g. proinflammatory activation). These processes contribute to the formation or intensification of symptoms of many diseases, including neurodegenerative conditions. There are reports of their involvement in the pathogenesis of Alzheimer's disease [31, 32], Parkinson's disease [33, 34], and amyotrophic lateral sclerosis [35].

Because the knowledge concerning the potential role of glycated proteins in the pathogenesis of MS is limited [36, 37], the aim of our study was to evaluate the intensity of the protein glycation process in patients with this disease. We hypothesised that MS is accompanied by an intensification of the protein glycation process expressed by elevated serum levels of selected AEGs (i.e. CML, CEL).

\section{Material and methods}

The study group consisted of 45 patients with MS (30 women and 15 men; mean age: $38.9 \pm 9.4$ years), who lived in the province of Silesia and were associated with SEZAM, which is the Silesian
Association of Multiple Sclerosis (Gliwice, Poland). The control group consisted of 31 healthy volunteers (20 women and 11 men; mean age: 42.4 \pm 11.3 years). Fasting blood samples were collected from the elbow vein. Serum obtained by centrifugation was stored at $-85^{\circ} \mathrm{C}$ until analysis.

Each MS patient completed the questionnaire related to their history (age, sex, place of residence, onset, duration, and course of the disease, degree of disability, quality of life, and treatment). The medical records were also analysed in detail. Each patient underwent neurological assessment, and the functional status was determined by the Expanded Disability Status Scale (EDSS). The in clusion criteria in the study group were as follows: age $\geq 18$ years, MS diagnosis based on McDonald's criteria (2010), the result of magnetic resonance imaging [38], and informed consent. The exclusion criteria were as follows: neurological comorbidities other than MS (i.e. dementia, previous stroke, neuropathy, cervical radiculopathy, etc.), chronic systemic diseases (i.e. diabetes, advanced heart failure, chronic renal disease, thyroid diseases, autoimmune diseases, etc.), and infectious diseases (especially Lyme disease). The control group consisted of healthy adults with no history of familial neurodegenerative diseases.

The research was approved by the Local Bioethics Committee of the Medical University of Silesia, Katowice. All participants were informed and gave their consent to participate in the study.

The concentrations of selected parameters of advanced protein glycation in serum samples were determined by enzyme-linked immunosorbent assay (ELISA) using commercially available kits. The carboxymethyllysine levels were measured using the OxiSelect N-(carboxymethyl) lysine (CML) Competitive ELISA Kit, and the carboxyethyllysine levels were measured using the OxiSelect N -(carboxyethyl)lysine (CEL) Competitive ELISA Kit (both from Cell Biolabs Inc., CA) according to the manufacturer's recommendations. Absorbances were read with the Power Wave XS plate reader (BioTek, Winooski, $\mathrm{VT}$ ) at $450 \mathrm{~nm}$ (reference wavelength $-630 \mathrm{~nm}$ ), and the results were processed with $\mathrm{KC}$ Junior software (BioTek, Winooski, VT). Determinations were performed during one series. The intraassay variation was below $8 \%$. The assay sensitivity was 2.25 $\mathrm{ng} / \mathrm{ml}$ for $\mathrm{CML}$ and $0.1 \mathrm{\mu g} / \mathrm{ml}$ for CEL.

\section{Statistical analysis}

The obtained results were presented using the basic parameters of descriptive statistics. The compatibility of distribution of variables with the normal distribution was checked by the Shapiro-Wilk test. Non-parametric Kolmogorov-Smirnov and $U$ Mann-Whitney tests were used for comparisons between the studied groups. The 
Kruskal-Wallis rank ANOVA test was used to study variability in the MS group. Spearman's rank test was used for correlations. $P<0.05$ was considered statistically significant. The calculations were performed using Statistica for Windows 12.0 (StatSoft, Cracow, Poland).

\section{Results}

The study group consisted of 45 patients with MS (67\% women, 33\% men), and the control group consisted of 31 healthy persons (65\% women, 35\% men). The gender distribution and age were comparable in both groups $(p=0.86$ and $p=$ 0.29 , respectively). The MS patients were characterised by different disease duration (from the first symptoms): $0-5$ years $-29 \%$; $6-10$ years $-33 \%$; $11-15$ years $-24 \%$; and 16 and more years $-14 \%$. Relapsing-remitting MS was diagnosed in $73 \%$ of patients, and secondary progressive MS in $27 \%$ of patients. The assessment of motor function according to the EDSS revealed that $40 \%$ of patients had scores ranging from 0 to 1.5 points, 33\% of patients from 2 to 4.5 points, and $27 \%$ of patients scores $\geq 5$ points. The most common symptoms of the disease in the study group included weakness in at least one limb (76\%), balance disorders (50\%), mood disorders (42\%), sensory disturbances (42\%), visual impairment (34\%), and bladder problems $(21 \%)$. Twenty patients were treated with disease-modifying drugs (DMD) (interferon $\beta$ -13 , glatiramer acetate -2 , natalizumab -2 , fingolimod -3 subjects).

The concentrations of CML and CEL in the serum of MS patients and controls are presented in Table I. No significant difference was found between men and women in both groups $(p>0.05$ for each comparison). Serum CML and CEL concentrations did not correlate with the age in the MS group $(R=-0.117 ; p=0.44$ for $C M L$ and $R=$ $0.026 ; p=0.86$ for $\mathrm{CEL})$ or in controls $(R=-0.169$; $p=0.36$ for $C M L$ and $R=-0.280 ; p=0.11$ for $C E L)$.
The mean serum concentration of CML was significantly higher in MS patients than in healthy subjects. The mean serum concentration of CEL was higher in the study group compared to the controls. However, the difference was not statistically significant (Table II).

Serum CML and CEL concentrations correlated positively in healthy controls $(R=0.50 ; p=0.004)$ but not in MS patients $(R=0.09 ; p=0.54)$. We found no association between the serum $C M L$ and CEL concentrations and the duration of the disease ( $p=0.60$ and $p=0.75$, respectively) and EDSS score ( $p=0.78$ and $p=0.81$, respectively) (Figures 1-4).

The mean CML and CEL concentrations were not statistically different between patients treated and untreated with immunomodulatory drugs ( $p=0.54$ and $p=1.00$, respectively). Moreover, the mean CML and CEL concentrations were similar in patients treated with interferon beta and those treated with other immunomodulatory drugs ( $p=$ 0.61 and $p=0.46$, respectively).

\section{Discussion}

The results of our study confirm the hypothesis that MS is associated with an intensification of the protein glycation process. We found significantly higher mean serum CML concentrations in MS patients than in healthy controls. The mean serum concentrations of CEL were also higher in MS subjects. However, this difference did not reach the threshold of statistical significance. Contrary to our results, Kalousova et al. [36] did not observe significant differences in total concentrations of AGEs or pentosidine in the cerebrospinal fluid and serum between MS and control groups. Additionally, they did not notice differences in mean concentrations of these parameters between patients with active and stable disease.

On the other hand, our results were partially consistent with the observations of Sternberg

Table I. CML and CEL concentrations in the serum of MS patients and healthy controls depending on gender (U Mann-Whitney test)

\begin{tabular}{|lcccccc|}
\hline \multirow{2}{*}{$\begin{array}{l}\text { Parameter } \\
\text { mean } \pm \text { SD) }\end{array}$} & \multicolumn{3}{c}{ MS patients $(n=45)$} & \multicolumn{3}{c|}{ Control group $(n=31)$} \\
\cline { 2 - 7 } & Men $(n=15)$ & Women $(n=30)$ & $P$-value & Men $(n=11)$ & Women $(n=20)$ & $P$-value \\
\hline CML $[\mathrm{ng} / \mathrm{ml}]$ & $26.55 \pm 8.17$ & $30.01 \pm 9.88$ & 0.219 & $21.81 \pm 8.89$ & $25.29 \pm 11.05$ & 0.432 \\
\hline CEL $[\mathrm{ng} / \mathrm{ml}]$ & $976.19 \pm 380.46$ & $1007.00 \pm 404.72$ & 0.791 & $699.09 \pm 381.90$ & $963.68 \pm 567.76$ & 0.252 \\
\hline
\end{tabular}

Table II. CML and CEL concentrations in the serum of MS patients and healthy controls ( $U$ Mann-Whitney test)

\begin{tabular}{|lccc|}
\hline $\begin{array}{l}\text { Parameter } \\
(\text { mean } \pm \text { SD })\end{array}$ & MS patients $(n=45)$ & Control group $(n=31)$ & $P$-value \\
\hline CML $[\mathrm{ng} / \mathrm{ml}]$ & $28.86 \pm 9.40$ & $24.06 \pm 10.33$ & 0.031 \\
\hline CEL $[\mathrm{ng} / \mathrm{ml}]$ & $996.73 \pm 392.72$ & $875.48 \pm 522.07$ & 0.201 \\
\hline
\end{tabular}




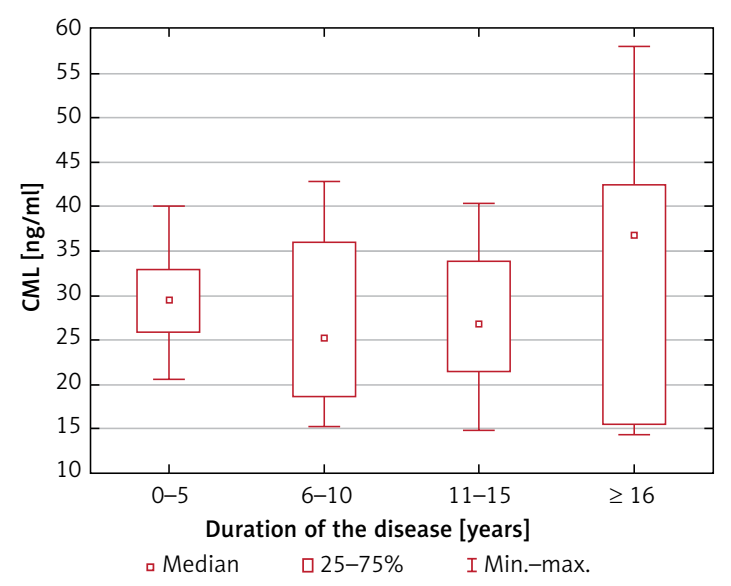

Figure 1. CML serum concentrations depending on the duration of MS (no statistically significant differences in the Kruskal-Wallis ANOVA rank test)

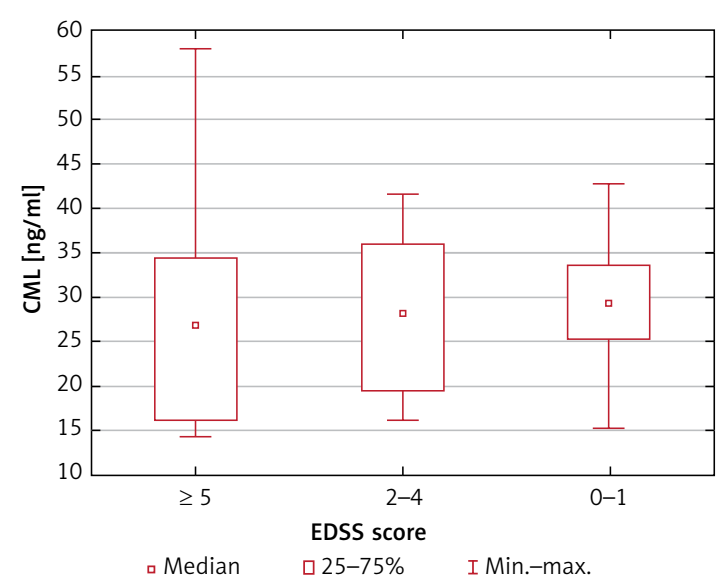

Figure 3. CML serum concentrations depending on the EDSS score (no statistically significant differences in the Kruskal-Wallis ANOVA rank test)

et al. [37], who also noticed the association between AGE concentration and MS. However, their results were contrary to ours. The mean CML concentration reported by Sternberg et al. was not significantly different between the MS group and healthy volunteers, while the mean serum CEL concentration was significantly higher in MS patients. Those authors concluded that AGEs, and particularly CEL, might be useful serum MS biomarkers and even suggested the introduction of AGE-inhibitor therapy [37]. Although the difference in CML concentrations between the groups did not reach the statistical significance threshold, the concentration of CML was higher in more than $45 \%$ of patients compared to the mean value in the control group. As regards CEL, the concentration of this parameter was higher in $91 \%$ of MS patients than the mean values in the controls. Similarly, higher CML and CEL concentrations were found in $69 \%$ and $47 \%$ of MS patients, respectively, compared to the mean values in the controls.

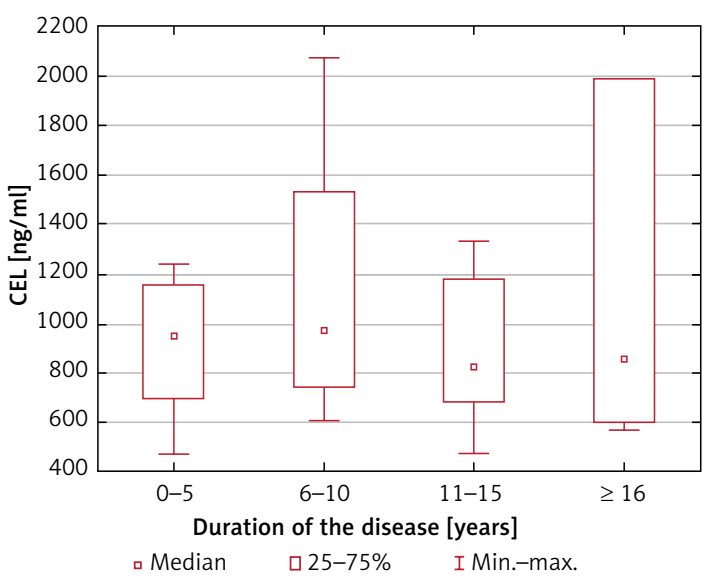

Figure 2. CEL serum concentrations depending on the duration of MS (no statistically significant differences in the Kruskal-Wallis ANOVA rank test)

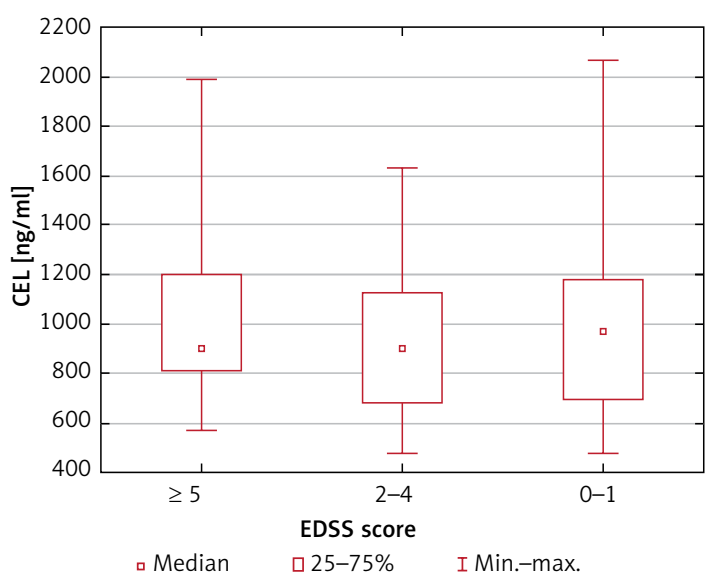

Figure 4. CEL serum concentrations depending on the EDSS score (no statistically significant differences in the Kruskal-Wallis ANOVA rank test)

The observations of Sternberg et al. [37] were different from our results in terms of the association between AGE concentration and gender. Both serum CML and CEL concentrations were significantly higher in healthy men than in healthy women. However, there were no gender differences in MS patients. In our study, no significant differences were found in MS or the control subjects. Sternberg et al. observed higher CML but not CEL concentrations in patients with active MS in comparison to patients with stable disease. In our study group, all MS patients were clinically stable, but serum CML concentrations were significantly higher compared to those in healthy subjects. Sternberg et al. [37] did not report any correlations between serum CML and CEL concentrations either in healthy subjects or in MS patients. In our study, both parameters correlated positively only in healthy controls. In MS patients no correlation was found between serum CML and CEL concentrations, which suggests the existence of addi- 
tional sources of CML in MS. However, our study revealed that neither $C M L$ nor $C E L$ concentrations differed significantly in relation to the degree of disability of patients, which is in line with the observations by Sternberg et al. [37]. Of note, the highest CML concentrations were observed in patients with an EDSS score of more than five points.

The presence of advanced protein glycation products may be associated with inflammation and increased oxidative stress [39]. Higher values of AGEs in MS patients may indicate a higher probability of disease relapse. The increase in plasma AGE concentrations in MS patients can probably be associated with dysfunction of the blood-brain barrier (BBB) [40] or may result from the activation of peripheral immune cells due to chronic inflammation and increased oxidative stress [41]. Although observations concerning the relationship between serum $C M L$ and $C E L$ levels and the incidence of MS are not clear, it should be noted that AGEs accumulate in the tissues (e.g. in the brain) and may indicate the ongoing inflammatory process. Wetzels et al. observed that AGE levels were increased in MS lesions due to the inflammatory activation of macrophages and astrocytes [30].

The determination of serum CML and CEL concentrations in the same patients during relapse and remission might provide better information on the utility of AGEs as markers for the risk of subsequent disease relapse [37].

In our study, we did not observe differences in concentrations of the examined parameters between patients treated and untreated with immunomodulatory agents. Sternberg et al. noticed that MS patients receiving immunomodulatory agents had lower mean serum CEL concentrations by $40 \%$ compared to untreated subjects, but they were still significantly higher than in the control group. Serum CML concentrations in both treated and untreated MS patients were comparable [37].

Other researchers examined the association between the receptor for advanced glycation end-products (RAGE) and disease-modifying drugs [42]. Rahimi et al. [43] and Asadikaram et al. [44] noticed elevated levels of serum soluble RAGE in patients treated with interferon $\beta$. Moreover, Sternberg et al. [45] observed that fingolimod also mediated modulation of the RAGE axis, which apparently contributed to the anti-inflammatory and neuroprotective effects of fingolimod. One-year treatment with this drug increased serum levels of soluble RAGE isoforms (i.e. sRAGE and esRAGE) by $32.4 \%$.

It was demonstrated that AGE levels were also higher in other neurological diseases [31-35]. In addition, AGEs are involved in many pathological processes in the nervous system. They affect my- elin proteins, which consequently are susceptible to phagocytosis by macrophages and contribute to demyelination and peripheral neuropathy, e.g. in patients with diabetes [46]. Immunohistochemical studies have also shown an increase in $\mathrm{CML}$ concentration in neurons and spinal cord microglia in patients with amyotrophic lateral sclerosis [47]. It has been proven that AGEs affect neurodegeneration (loss of neurons), which not only occurs in the course of diseases commonly considered neurodegenerative, such as Alzheimer's disease or Parkinson's disease, but it is also evident in the development of inflammatory and demyelinating diseases of the brain and the spinal cord, such as MS [48-50]. The pathomechanism of neurodegeneration in MS is not fully understood [51]. Wetzels et al. hypothesised that AGEs could accelerate the occurrence of MS lesions by inducing a proinflammatory activity in microglia and astrocytes. It is also associated with increased RAGE expression. Moreover, AGEs could destroy the BBB function, which leads to the increased infiltration of peripheral immune cells into the central nervous system, leading to neuroinflammation and neurodegeneration [52, 53].

Polymorphism in glutathione transferase-encoding genes was found in MS patients. It leads to a decrease in detoxification and antioxidant potential $[54,55]$. However, an increase in the concentration of malonic dialdehyde and a decrease in glutathione peroxidase activity were observed in the cerebrospinal fluid of MS patients $[56,57]$. Neurons are particularly sensitive to oxidative disorders due to the increased aerobic metabolism, a high content of unsaturated fatty acids, and relatively low levels of (enzymatic and non-enzymatic) antioxidants. Even short-term oxygen deficiency results in an increase in the concentration of reactive oxygen species and damage to lipids, proteins, and DNA [58]. Hence, a variety of oxidative stress markers have been sought in MS [59].

In conclusion, it should be stressed that the issue of the involvement of the glycation process in the aetiopathogenesis of MS remains open. There is still little research related to this subject, and the results of studies are inconclusive and often contradictory. This study should be another attempt to clarify whether and to what extent post-translational protein processing plays a role in the incidence, course, and severity of MS. It appears that the disease is accompanied by an increase in the glycation processes, especially in metabolic pathways leading to carboxymethyllysine formation. However, it is not possible to decide whether it is the cause or the effect of inflammation. The course of MS also changes some of the relationships between different glycation products, which may suggest additional sources of their forma- 
tion during the disease process. Further research is warranted to clarify whether and which AGEs could serve as diagnostic and therapeutic biomarkers for MS; it is of great interest, especially in the context of new medicinal implications and the suggestion for the introduction of AGE-inhibitors to the therapy of MS. These inhibitors have also been proposed as promising neuroprotective compounds [60]. It seems that these actions combined with multidisciplinary rehabilitation could improve the quality of life in patients with MS [61].

Multiple sclerosis is accompanied by an intensification of protein glycation process, especially within the pathways leading to the formation of carboxymethyllysine. The duration of the disease and the degree of disability seem not to affect the progression of the glycation process. However, the disease process associated with MS may affect the relationship between CML and CEL concentrations.

The small size of patient groups and subgroups (i.e. treated and untreated with DMD) could influence statistical differences. We examined only clinically stable patients. However, it would be interesting to compare serum CEL and CML levels during relapse and remission in the same patients. Therefore, further studies are warranted in this respect.

\section{Acknowledgments}

This study was supported by a research grant from the KNW-1-148/K/8/0 of SUM in Katowice.

\section{Conflict of interest}

The authors declare no conflict of interest

\section{References}

1. Pirko I, Noseworthy JH. Demyelinating disorders of the central nervous system. In: Textbook of Clinical Neurology. $3^{\text {rd }}$ edn. Elsevier Inc, Amsterdam 2007; 1103-33.

2. Giorgio A, Battaglini M, Smith SM, De Stefano N. Brain atrophy assessment in multiple sclerosis: importance and limitations. Neuroimaging Clin N Am 2008; 18: 675-86.

3. Bermejo PE, Oreja-Guevara C, Diez-Tejedor E. Pain in multiple sclerosis: prevalence, mechanisms, types and treatment. Rev Neurol 2010; 50: 101-8.

4. Flachenecker P, Kumpfel T, Kallmann B, et al. Fatigue in multiple sclerosis: a comparison of different rating scales and correlation to clinical parameters. Mult Scler 2002; 8: 523-6.

5. Martin R, Hohlfeld R, McFarland HF. Multiple sclerosis. In: Brandt T, Caplan LR, Dichgans J, Diener HC, Kennard C: Neurological Disorders Course and Treatment. $2^{\text {nd }}$ edn. Academic Press, Cambridge 2003; 677-9.

6. Gilden DH. Infectious causes of multiple sclerosis. Lancet Neurol 2005; 4: 195-202.

7. Correale J, Farez M.The impact of parasite infections on the course of multiple sclerosis. J Neuroimmunol 2011; 233: 6-11.
8. Ebers GC. Genetics and multiple sclerosis. An Neurol 1994; 36: 12-6.

9. Zhang $X$, Tang Y, Sujkowska D. Degenerate TCR recognition and dual DR2 restriction of autoreactive T cells: implications for the initiation of the autoimmune response in multiple sclerosis. Eur J Immunol 2008; 38: 1297-309.

10. Markovic-Plese S. Degenerate T-cell receptor recognition, autoreactive cells and the autoimmune response in multiple sclerosis. Neuroscientist 2009; 15: 225-31.

11. Antel J, Bar-Or A. Roles of immunoglobulins and B cells in multiple sclerosis: from pathogenesis to treatment. J Neuroimmunol 2006; 180: 3-8.

12. Disanto G, Morahan JM, Barnett MH. The evidence for a role of B cells in multiple sclerosis. Neurology 2012; 78: 823-32

13. Scazzone C, Agnello L, Ragonese P, et al. Association of CYP2R1 rs 10766197 with MS risk and disease progression. J Neurosci Res 2018; 96: 297-304.

14. Ciaccio M, Fugardi G, Titone L, et al. Procalcitonin levels in plasma in oncohaematologic patients with and without bacterial infections. Clin Chim Acta 2004; 340: 149-52.

15. Qing-Feng S, Ying-Peng X, Tian-Tong X. Matrix metalloproteinase- 9 and $p 53$ involved in chronic fluorosis induced blood-brain barrier damage and neurocyte changes. Arch Med Sci 2019; 15: 457-66.

16. Bivona G, Gambino CM, Iacolino G, Ciaccio M. Vitamin D and the nervous system. Neurol Res 2019; 41: 827-35.

17. Bivona G, Agnello L, Bellia C, et al. Non-skeletal activities of vitamin $D$ : from physiology to brain pathology. Medicina (Lithuania) 2019; 55: 341.

18. Bivona G, Agnello L, Ciaccio M. The immunological implications of the new Vitamin D metabolism. Cent Eur J Immunol 2018; 43: 331-4.

19. Scazzone C, Agnello L, Lo Sasso B, et al. Vitamin D and multiple sclerosis: an open-ended story. Open Biochem J 2019; 13: 88-98.

20. Agnello L, Scazzone C, Lo Sasso B, et al. VDBP, CYP27B1, and 25-hydroxyvitamin $\mathrm{D}$ gene polymorphism analyses in a group of Sicilian multiple sclerosis patients. Biochem Genet 2017; 55: 183-92.

21. Tessier FJ. The Maillard reaction in human body. The main discovers and factors that affect glycation. Pathol Biol 2010; 58: 214-9.

22. Basta G. Receptor for advanced glycation endproducts and atherosclerosis: from basic mechanisms to clinical implications. Atherosclerosis 2008; 196: 9-21.

23. Yan SF, Ramasamy R, Schmidt AM. Mechanisms of disease: advanced glycation endproducts and their receptor in inflammation and diabetes complications. Nat Clin Pract Endocrinol Metab 2008; 4: 285-93.

24. Smith KJ, Kapoor R, Felts PA. Demyelination: the role of reactive oxygen and nitrogen species. Brain Pathol 1999; 9: 69-92.

25. Singh R, Barden A, Mori T, Beilin L. Advanced glycation end-products: a review. Diabetologia 2001; 44: 129-46.

26. Tsukahara H, Sekine K, Uchiyama M, et al. Formation of advanced glycosylation end products and oxidative stress in young patients with type 1 diabetes. Pediatr Res 2003; 54: 419-24.

27. Teerlink T, Barto R, Ten Brink HJ, Schalkwijk CG. Measurement of $\mathrm{Ne}$-(carboxymethyl)-lysine and $\mathrm{Ne}$-(carboxyethyl)-lysine in human plasma protein by stable-isotope-dilution tandem mass spectrometry. Clin Chem 2004; 50: 1222-8.

28. Urlich P, Cerami A. Protein glycation, diabetes and aging. Recent Prog Horm Res 2001; 56: 1-21. 
29. Ying C, Wang S, Lu Y, et al. Glucose fluctuation increased mesangial cell apoptosis related to AKT signal pathway. Arch Med Sci 2019; 15: 730-7.

30. Wetzels S, Vanmierlo T, Scheijen JLJM, et al. Methylglyoxal-derived advanced glycation endproducts accumulate in multiple sclerosis lesions. Front Immunol 2019; 10: 855 .

31. Leszek J, Małyszczak K, Bartys A, Staniszewska M, Gamian A. Analysis of serum patients with Alzheimer's disease for the level of advanced glycation end products. Am J Alzheimers Dis Other Demen 2006; 21: 1-6.

32. Takeuchi M, Yamagishi S. Possible involvement of advanced glycation end-products (AGEs) in the pathogenesis of Alzheimer's disease. Curr Pharm Des 2008; 14: 973-8.

33. Salahuddin P, Rabbani G, Khan RH. The role of advanced glycation end products in various types of neurodegenerative disease: a therapeutic approach. Cell Mol Biol Lett 2014; 19: 407-37.

34. Vicente Miranda H, El-Agnaf OM, Outeiro TF. Glycation in Parkinson's disease and Alzheimer's disease. Mov Disord 2016; 31: 782-90.

35. Ilzecka J. Serum-soluble receptor for advanced glycation end product levels in patients with amyotrophic lateral sclerosis. Acta Neurol Scand 2009; 120: 119-22.

36. Kalousova M, Havrdova E, Mrazova K, Specek P, Braun M. Advanced glycoxidation end products in patients with multiple sclerosis. Prague Med Rep 2005; 106: 167-74.

37. Sternberg Z, Hennies C, Sternberg D, et al. Diagnostic potential of plasma carboxymethyllysine and carboxyethyllysine in multiple sclerosis. J Neuroinflammation 2010; 7: 72.

38. Polman CH, Reingold SC, Banwel B. Diagnostic criteria for multiple sclerosis: 2010 revisions to the "McDonald criteria". Ann Neurol 2011; 69: 292-302.

39. Miranda HV, Outeiro TF. The sour side of neurodegenerative disorders: the effects of protein glycation. J Patho 2010; 221: 13-25.

40. Weiss N, Miller F, Cazaubon S, Couraud PO. The bloodbrain barrier in brain homeostasis and neurological diseases. Biochem Biophys Acta 2009; 1788: 842-57.

41. Thornalley PJ, Battah S, Ahmed N, et al. Quantitative screening of advanced glycation endproducts in cellular and extracellular proteins by tandem mass spectrometry. Biochem I 2003; 375: 581-92.

42. Sternberg Z, Sternberg D, Drake A, Chichelli T, Yu J, Hojnacki D. Disease modifying drugs modulate endogenous secretory receptor for advanced glycation end-products, a new biomarker of clinical relapse in multiple sclerosis. J Neuroimmunol 2014; 274: 197-201.

43. Rahimi M, Aghabozorg Afjeh SS, Omrani MD, et al. Soluble receptor for advanced glycation end products (sRAGE) is up-regulated in multiple sclerosis patients treated with interferon beta-1a. Cell Physiol Biochem 2018; 46: 561-7.

44. Asadikaram G, Noroozi S, Ebrahimi Meimand HA, et al. Interferon-beta 1a modulates expression of RAGE but not S100A12 and nuclear factor-kappaB in multiple sclerosis patients. Neuroimmunomodulation 2016; 23: 345-51.

45. Sternberg Z, Kolb C, Chadha K, et al. Fingolimod anti-inflammatory and neuroprotective effects modulation of RAGE axis in multiple sclerosis patients. Neuropharmacology 2018; 130: 71-6.

46. Turk Z. Glycotoxines, karbonyl stress and relevance to diabetes and its complications. Physiol Res 2010; 59: 147-56.
47. Juranek J, Daffu G, Wojtkiewicz J, Lacomis D, Kofler J, Schmidt AM. Receptor for advanced glycation end products and its inflammatory ligands are upregulated in amyotrophic lateral sclerosis. Front Cell Neurosci 2015; 9: 485.

48. Li J, Liu D, Sun L, Lu Y, Zhang Z. Advanced glycation end products and neurodegenerative diseases: mechanisms and perspective. J Neurol Sci 2012; 317: 1-5.

49. Salahuddin P, Rabbani G, Khan RH. The role of advanced glycation end products in various types of neurodegenerative disease: a therapeutic approach. Cell Mol Biol Lett 2014; 19: 407-37.

50. Deluca GC, Ebers GC, Esiri MM. Axonal loss in multiple sclerosis: a pathological survey of the corticospinal and sensory tracts. Brain 2004; 127: 1009-18.

51. Wolinski P, Jałosinski M, Głąbinski A. Mechanisms of neurodegeneration and its markers in multiple sclerosis. Aktualn Neurobiol 2008; 1: 25-32.

52. Wetzels S, Wouters K, Schalkwijk CG, Vanmierlo T, Hendriks JJA. Methylglyoxal-derived advanced glycation endproducts in multiple sclerosis. Int J Mol Sci 2017; 18: E421.

53. Wetzels S, Wouters K, Miyata T, et al. Advanced glycation endproducts are increased in the animal model of multiple sclerosis but cannot be reduced by pyridoxamine treatment or glyoxalase 1 overexpression. Int J Mol Sci 2018; 19: E1311.

54. Stavropoulou C, Korakaki D, Rigana H, et al. Glutathione-S-transferase $\mathrm{T} 1$ and $M 1$ gene polymorphisms in Greek patients with multiple sclerosis: a pilot study. Eur J Neurol 2007; 14: 572-4.

55. Parchami Barjui S, Reiisi S, Bayati A. Human glutathione $\mathrm{s}$-transferase enzyme gene variations and risk of multiple sclerosis in Iranian population cohort. Mult Scler Relat Disord 2017; 17: 41-6.

56. Calabrese V, Raffaele R, Cosentino E, Rizza V. Changes in cerebrospinal fluid levels of malondialdehyde and glutathione reductase activity in multiple sclerosis. Int J Clin Pharmacol Res 1994; 14: 119-23.

57. Bartova R, Petrlenicova D, Oresanska K, et al. Changes in levels of oxidative stress markers and some neuronal enzyme activities in cerebrospinal fluid of multiple sclerosis patients. Neuro Endocrinol Lett 2016; 37: 102-6.

58. Kopczynska E, Torlinski L, Ziołkowski M. The impact of alcohol addiction on the parameters of oxidative stress. Postepy Hig Med Dosw 2001; 55: 95-111.

59. Ibitoye R, Kemp K, Rice C, Hares K, Scolding N, Wilkins A. Oxidative stress-related biomarkers in multiple sclerosis: a review. Biomark Med 2016; 10: 889-902.

60. Munch G, Deuther-Conrad W, Gasic-Milenkovic J. Glycoxidative stress creates a vicious cycle of neurodegeneration in Alzheimer's disease - a target for neuroprotective treatment strategies? J Neural Transm Suppl 2002; 62: 303-7.

61. Nedeljkovic U, Dackovic J, Tepavcevic Kisic D, et al. Multidisciplinary rehabilitation and steroids in the management of multiple sclerosis relapses: a randomized controlled trial. Arch Med Sci 2016; 12: 380-90. 